\title{
A unified bijective method for maps: application to two classes with boundaries
}

\author{
Olivier Bernardi $\|^{\|}$and Éric Fusy $\|^{2}$ \\ ${ }^{1}$ Department of Mathematics, MIT, 77 Massachusetts Avenue, Cambridge MA 02139, USA. \\ ${ }^{2}$ LIX, École Polytechnique, 91128 Palaiseau Cedex, France.
}

\begin{abstract}
Based on a construction of the first author, we present a general bijection between certain decorated plane trees and certain orientations of planar maps with no counterclockwise circuit. Many natural classes of maps (e.g. Eulerian maps, simple triangulations,...) are in bijection with a subset of these orientations, and our construction restricts in a simple way on the subset. This gives a general bijective strategy for classes of maps. As a nontrivial application of our method we give the first bijective proofs for counting (rooted) simple triangulations and quadrangulations with a boundary of arbitrary size, recovering enumeration results found by Brown using Tutte's recursive method.

Résumé. En nous appuyant sur une construction du premier auteur, nous donnons une bijection générale entre certains arbres décorés et certaines orientations de cartes planaires sans cycle direct. De nombreuses classes de cartes (par exemple les eulériennes, les triangulations) sont en bijection avec un sous-ensemble de ces orientations, et notre construction se spécialise de manière simple sur le sous-ensemble. Cela donne un cadre bijectif général pour traiter les familles de cartes. Comme application non-triviale de notre méthode nous donnons les premières preuves bijectives pour l'énumération des triangulations et quadrangulations simples (enracinées) ayant un bord de taille arbitraire, et retrouvons ainsi des formules de comptage trouvées par Brown en utilisant la méthode récursive de Tutte.
\end{abstract}

Keywords: Triangulation, quadrangulation, maps with boundaries, mobiles, bijection, counting

\section{Introduction}

The enumeration of planar maps (connected graphs embedded on the sphere) has received a lot of attention since the seminal work of Tutte in the 60's [Tut63]. Tutte's recursive method consists in translating the decomposition of a class of maps (typically obtained by deleting an edge) into a functional equation satisfied by the corresponding generating function. The translation usually requires an additional "catalytic" variable, and the obtained functional equation is solved using the so-called "quadratic method" GJ83, sec.2.9] or its extensions [BMJ06]. The final result is, for many classes of maps, a strikingly simple counting formula. For instance, the number of (rooted) maps with $n$ edges is $\frac{2 \cdot 3^{n}}{(n+1)(n+2)}\left(\begin{array}{c}2 n \\ n\end{array}\right)$. Tutte's

\footnotetext{
${ }^{\dagger}$ Supported by the French ANR project A3 and the European project ExploreMaps - ERC StG 208471

${ }^{\ddagger}$ Supported by the European project ExploreMaps - ERC StG 208471

1365-8050 @ 2010 Discrete Mathematics and Theoretical Computer Science (DMTCS), Nancy, France
} 
method has the advantage of being systematic, but is quite technical in the way of solving the equation and does not give a combinatorial understanding of the simple-looking enumerative formulas.

As an alternative method, bijective constructions have been developed to obtain more direct combinatorial proofs of the counting formulas, with nice algorithmic applications (random generation and asymptotically optimal encoding in linear time). The first bijections appeared in [CV81] and later in [Sch98] where direct bijections for several classes of maps are described. Typically bijections are from a class of "decorated" plane trees to a class of maps and operate on trees by progressively closing facial cycles. Even if it has been successfully applied to many classes, e.g. in [PS06, PS03, FPS08, BFG04], the bijective method for maps is up to now not as systematic as Tutte's recursive method, since for each class of maps one has to "guess" the tree family to match with, and one has to specify the construction from trees to maps.

This article contributes to fill this gap. Based on a construction of the first author [Ber07, BC10], we provide in Section 3 a general bijection $\Phi$ between a set $\mathcal{D}$ of certain decorated plane trees which we call mobile ${ }^{(i)}$ and a set $\mathcal{O}$ of certain orientations on planar maps with no counterclockwise circuit. As it turns out, a map class is often in bijection with a subfamily $\mathcal{S}$ of $\mathcal{O}$ on which our construction restricts nicely; typically the orientations in $\mathcal{S}$ are characterized by degree constraints which can be traced through our construction and yields a degree characterization of the associated mobiles. The mobiles family is then specifiable by a decomposition grammar and amenable to the Lagrange inversion formula for counting. To summarize, our method makes the bijective method more systematic, since it consists in specializing a "master bijection" $\Phi$ to the class of maps under consideration. The problem of enumerating a class of map $\mathcal{M}$ therefore reduces to guessing a family of "canonical" orientations (in $\mathcal{O}$ ) for $\mathcal{M}$ instead of guessing a family of trees to match with $\mathcal{M}$ (the first task being often simpler than the second).

We focus here, in Section 4 and Section 5 respectively, on two classes that were not completely covered before, namely simple triangulations and simple quadrangulations with a polygonal boundary and a rootcorner incident to the boundary. We show bijectively that the number $t_{n, k}$ of rooted simple triangulations with $n+k$ vertices and boundary of length $k$ and the number $q_{n, k}$ of rooted simple quadrangulations with $n+2 k$ vertices and boundary of length $2 k$ satisfy

$$
t_{n}^{(k)}=\frac{2(2 k-3) !}{(k-1) !(k-3) !} \frac{(4 n+2 k-5) !}{n !(3 n+2 k-3) !}, \quad q_{n}^{(k)}=\frac{3(3 k-2) !}{(k-2) !(2 k-1) !} \frac{(3 n+3 k-4) !}{n !(2 n+3 k-2) !},
$$

recovering results found by Brown respectively in [Bro64] and [Bro65] using Tutte's recursive method. The case without boundaries ( $k=3$ for triangulations, $k=2$ for quadrangulations) have already received bijective proofs in [PS06, FPS08] (for triangulations) and [Fus07, Sch98] (for quadrangulations); our construction actually coincides with [FPS08, Theo.4.10] for triangulations and with [Sch98, Sec.2.3.3] for quadrangulations. The case of triangulations with boundaries has also received a partial bijective interpretation, different from ours, in [PS06] (only one direction is given, from trees to maps, which by injection shows that $t_{n, k}$ is at least the number above, but does not suffice to prove equality).

\section{Maps and orientations}

Maps. A (planar) map is a connected planar graph embedded in the oriented sphere and considered up to continuous deformation. A map is simple if it has no loop nor multiple edge. The faces are the connected components of the complementary of the graph. A plane tree is a map with a unique face. Cutting an

(i) The term mobile is borrowed from a bijection by Bouttier et al. [BFG04] which can be seen as a specialization of $\Phi$. 
edge $e$ at its middle point gives two half-edges, each incident to an endpoint of $e$ (they are both incident to the same vertex if $e$ is a loop). We shall also consider some maps decorated with dangling half-edges called stems (see e.g. Figure 2(a)). A corner is the angular section between two consecutive half-edges around a vertex. The degree of a vertex or face is the number of incident corners. A map is a triangulation (resp. quadrangulation) if every face has degree 3 (resp. 4).

A map is said to be vertex-rooted if a vertex is marked, face-rooted if a face is marked, and cornerrooted if a corner is marked(ii). The marked vertex, face or corner are called the root-vertex, root-face or root-corner. For a corner-rooted map, the marked corner is indicated by a dangling half-edge pointing to that corner; see Figure 1. A corner-rooted map is said to induce the vertex-rooted map (resp. facerooted map) obtained by keeping the root-vertex (resp. root-face) as marked, but otherwise forgetting the root-corner. Given a face-rooted (or corner-rooted) map, vertices and edges are said to be outer or inner depending on whether they are incident to the root-face or not.

Orientations. An orientation $O$ of a map $M$ is the choice of a direction for each edge of $M$. A circuit is a directed cycle. A source is a vertex incident to no ingoing edge. If $M$ is face-rooted (resp. vertex-rooted, corner-rooted), then the pair $(M, O)$ is called a face-rooted orientation (resp. vertex-rooted orientation, corner-rooted orientation). A corner-rooted orientation naturally induces a face-rooted orientation and a vertex-rooted orientation. For a vertex $v$ of $M$, the indegree in $(v)$ is the number of edges going into $v$; the outdegree $\operatorname{out}(v)$ is the number of edges going out of $v$. For a face $f \in M$, the clockwise-degree $\operatorname{cw}(f)$ is the number of edges incident to $f$ that have $f$ on their right; the counterclockwise-degree ccw $(f)$ is the number of edges that have $f$ on their left. For corner-rooted maps, the half-edge indicating the root-corner increases by 1 the indegree of the root-vertex and the clockwise-degree of the root-face.

A vertex-rooted orientation is said to be accessible if every vertex is accessible from the root-vertex by a directed path; it is source-accessible if in addition the root-vertex is a source. A circuit of a face-rooted (or corner-rooted) orientation is said clockwise if the root-face is on its left. The orientation is minimal if every circuit is clockwise; it is clockwise-minimal if in addition the root-face is a (clockwise) circuit. We extend the definition of accessibility to (face-rooted) clockwise-minimal orientations $O$ by calling $O$ accessible if it is accessible from one of the vertices incident to the root-face. Observe that $O$ is in fact accessible from any vertex on the root-face in this case Similarly, we call a source-accessible orientation $O$ minimal if $O$ is minimal for one of the faces incident to the root-vertex. Observe that $O$ is in fact minimal for every face incident to the root-vertex in this case.

Let $d$ be a positive integer. We denote by $\mathcal{S}_{d}$ the set of source-accessible minimal orientations such that the root-vertex has degree $d$. We denote by $\mathcal{O}_{d}$ the set of clockwise-minimal accessible orientations such that the root-face has degree $d$. We denote by $\widetilde{\mathcal{S}}_{d}$ the subset of $\mathcal{S}_{d}$ such that every face incident to the root-vertex has clockwise degree 1 . We denote by $\widetilde{\mathcal{O}}_{d}$ the subset of $\mathcal{O}_{d}$ such that every vertex incident to the root-face has indegree 1.

Given a map $M$ with vertex-set $V$ and given a function $\alpha: V \mapsto \mathbb{N}$, an $\alpha$-orientation is an orientation of $M$ such that in $(v)=\alpha(v)$ for each $v \in V$. The following result is well-known [Fel04]:

Lemma 1 If a face-rooted map $M$ has an $\alpha$-orientation, then $M$ has a unique minimal $\alpha$-orientation.

Duality. The $d u a l M^{*}$ of a map $M$ is the map obtained by the following two step process; see Figure 1

(ii) Corner-rooted map are usually simply called rooted maps in the literature. A face-rooted map can be thought as a plane map (a connected graph embedded in the plane) by thinking of the root-face as the infinite face. 

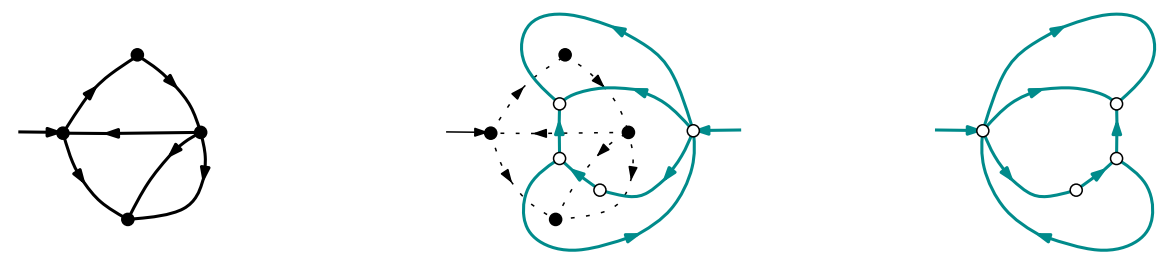

Fig. 1: The dual of an oriented map.

1. In each face $f$ of $M$, draw a vertex $v_{f}$ of $M^{*}$. For each edge $e$ of $M$ separating faces $f$ and $f^{\prime}$ (which can be equal), draw the dual edge $e^{*}$ of $M^{*}$ going from $v_{f}$ to $v_{f^{\prime}}$ across $e$.

2. Flip the drawing of $M^{*}$, that is, inverse the orientation of the sphere.

The dual of a face-rooted map is a vertex-rooted map. Corners of a map and of its dual are in natural correspondence (they face each other); this gives the way of defining the root-corner of the dual of a corner-rooted map; see Figure 1. Duality is involutive on maps and rooted maps.

The dual of an orientation of $M$ is the orientation of the dual map $M^{*}$ obtained by applying the following rule at step 1: the dual-edge $e^{*}$ of an edge $e \in M$ is oriented from the left of $e$ to the right of $e$. Observe that duality is an involution for oriented map (this is the motivation for step 2 in the definition of duality). The clockwise degree (resp. counterclockwise degree) of a face $f$ of $M$ is equal to the indegree (resp. outdegree) of the vertex $v_{f}$ of $M^{*}$ (this is true also with the special convention applying to corner-rooted maps). Also one easily checks that minimality is equivalent to accessibility in the dual:

Lemma 2 A face-rooted orientation is minimal (resp. clockwise-minimal) if and only if the dual vertexrooted orientation is accessible (resp. source-accessible).

Observe that duality maps the set of orientations $\mathcal{S}_{d}$ (resp. $\widetilde{\mathcal{S}}_{d}$ ) to the set $\mathcal{O}_{d}$ (resp. $\widetilde{\mathcal{O}}_{d}$ ). Also, minimal accessible orientations (of corner-rooted maps) are self dual. We mention that these orientations, which play an important role below, are in bijection with spanning trees [Ber07].

\section{Bijections between mobiles and orientations}

In this section, we first recall a bijection $\Phi$ originally due to the first author [Ber07]. We then present some extensions of $\Phi$ which will be convenient for our subsequent goals. Indeed, in the next two sections we will show how to use these extensions in order to count several families of maps.

The bijection $\Phi$ maps minimal accessible (corner-rooted) orientations with $n$ edges and pairs of cornerrooted plane trees $(B, T)$ with $n+1$ and $n$ edges respectively. The tree $B$ is called the (rooted) mobile and its vertices are bicolored in black and white (in such a way that edges always connect a black and a white vertex). Informally, the bijection $\Phi$ consists in folding the tree $T$ (oriented from the root to leaves) around the mobile. More precisely, one glues the vertices of $T$ on the black corners of the mobile and then erases the edges and white vertices of $B$ (leaving the edges of $T$ as edges of a minimal accessible orientation). In what follows we adopt a slightly different presentation, in which the tree $T$ only appears implicitly in certain decorations added to the mobile $B$.

A decorated mobile is a bicolored (unrooted) plane tree with outgoing stems (dangling outgoing halfedges) possibly attached to each black corner; see Figure 2(a). The excess of a decorated mobile is the number of edges minus the number of (outgoing) stems. A mobile with excess $\delta$ is called a $\delta$-mobile. A fully decorated mobile is obtained from a decorated mobile by inserting an ingoing stem (dangling ingoing 


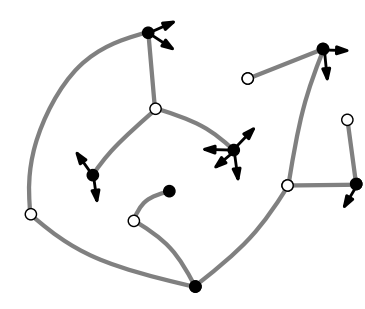

(a)

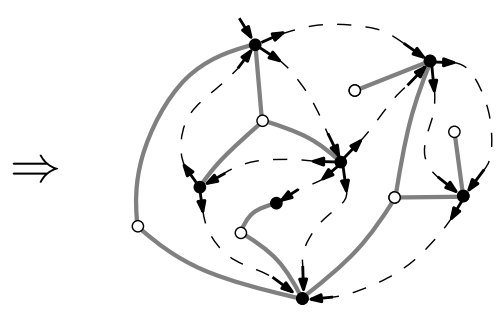

(b)

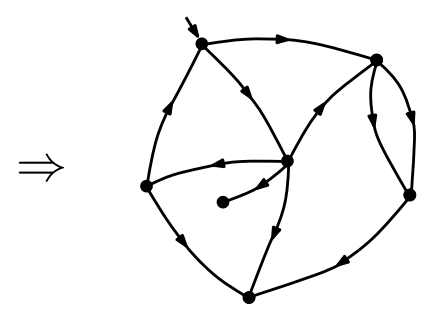

(c)

Fig. 2: The rooted closure of a mobile of excess $\delta=1$.

half-edge) in each black corner following an edge of the mobile (and not a stem) in clockwise order around the vertex; the fully decorated mobile is represented in solid lines in Figure 2(b). The degree deg $(v)$ of a vertex $v$ of a decorated mobile is the total number of incident half-edges (including the outgoing stems). For a black vertex $b$ the indegree in $(b)$ and out-degree out $(b)$ are respectively the number of incident ingoing and outgoing stems incident to $b$ in the fully-decorated mobile (so $\operatorname{deg}(b)=i n(b)+\operatorname{out}(b)$ ).

\subsection{Bijection between 1-mobiles and minimal accessible orientations}

We now recall the bijection given in [Ber07] between 1-mobiles and minimal accessible orientations.

Closure. Let $D$ be a decorated mobile with $p$ edges and $q$ outgoing stems (hence excess $\delta=p-q$ ). The corresponding fully decorated mobile $D^{\prime}$ has $p$ ingoing and $q$ outgoing stems. A clockwise walk around $D^{\prime}$ (with the face area on the left of the walker) sees a succession of outgoing stems and ingoing stems. Associating an opening parenthesis to outgoing stems and a closing parenthesis to ingoing stems, one obtains thus a cyclic binary word with $q$ opening and $p$ closing parentheses. This yields in turn a matching of outgoing stems with ingoing stems, leaving $|\delta|$ stems unmatched, which are ingoing if $\delta>0$ and outgoing if $\delta<0$; see Figure 2 The partial closure $C$ of the decorated mobile $D$ is obtained by forming a directed edge out of each matched pair, see Figure 2 a)-(b). We consider $C$ as a planar map with two types of edges (those of the mobile, which are undirected, and the new formed edges, which are directed) and $|\delta|$ stems. Note that, if $\delta \geq 0$, there are $\delta$ white corners incident to the root-face of $C$, because initially the number of such corners is equal to the number of edges, and then each matched pair of stems decreases this number by 1 . These corners, which stay incident to the root-face throughout the partial closure, are called exposed white corners.

The rooted-closure of the decorated mobile $D$ is obtained from the partial closure $C$ by erasing every white vertex and edge of the mobile (this might result in a disconnected embedded graph in general).

Opening. Let $M$ be an oriented map (rooted or not) with vertex set $V$ and face set $F$. The partial opening of $M$ is the map $C$ with two types of vertices (black vertices in $V$ and white vertices in $W=\left\{w_{f}, f \in\right.$ $F\}$ ) and two types of edges (directed and undirected) obtained as follows.

- Insert a white vertex $w_{f}$ inside each face $f$ of $M$.

- Draw an undirected edge between $w_{f}$ and each corner incident to $f$ which precedes an ingoing halfedge in clockwise order around its incident vertex. If $M$ is corner-rooted, then the stem indicating the root-corner is interpreted as an ingoing half-edge and gives rise to an edge of $C$.

If $M$ is a corner-rooted orientation, the rooted-opening of $M$ is obtained from the partial opening $C$ by 


\begin{tabular}{|c|c|c|c|c|}
\hline \multirow[t]{3}{*}{ Rooted closure, duality: } & 1-mobile & \multicolumn{2}{|c|}{ max. acc. ori. } & max. acc. ori. \\
\hline & black vertex $b$ & $\underset{\begin{array}{c}\operatorname{deg}(b)=\operatorname{deg}(v) \\
\text { in }(b)=\operatorname{in}(v) \\
\operatorname{out}(b)=\operatorname{out}(v)\end{array}}{\longrightarrow}$ vertex & 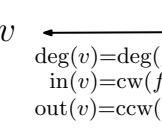 & $\begin{array}{l}\overrightarrow{(f)} \\
f() \\
(f)\end{array}$ \\
\hline & white vertex $w$ & $\underset{\operatorname{deg}(w)=\operatorname{cw}(f)}{\longrightarrow}$ face $f$ & $\overleftarrow{\operatorname{cw}(f)=\operatorname{in}(v}$ & $\overrightarrow{v)} \quad$ vertex $v$ \\
\hline \multirow[t]{6}{*}{$\delta$-closure + duality: } & \multirow{3}{*}{ Case $\delta>0$} & $\delta$-mobile & & ori. in $\mathcal{O}_{\delta}$ \\
\hline & & black vertex $b$ & $\begin{aligned} \stackrel{\operatorname{deg}(b)=\operatorname{deg}(f)}{\text { in }(b)=\operatorname{cw}(f)} \\
\operatorname{out}(b)=\operatorname{cow}(f)\end{aligned}$ & inner face $f$ \\
\hline & & white vertex $w$ & $\overleftrightarrow{\operatorname{deg}(w)=\operatorname{in}(v)}$ & vertex $v$ \\
\hline & \multirow{3}{*}{ Case $\delta<0$} & $\delta$-mobile & & ori. in $\widetilde{\mathcal{O}}_{|\delta|}$ \\
\hline & & black vertex $b$ & $\begin{aligned} \stackrel{\operatorname{deg}(b)=\operatorname{deg}(f)}{\text { in }(b)=\operatorname{cw}(f)} \\
\operatorname{out}(b)=\operatorname{ccw}(f)\end{aligned}$ & inner face $f$ \\
\hline & & white vertex $w$ & $\underset{\operatorname{deg}(w)=\operatorname{in}(v)}{\longrightarrow} \mathrm{i}$ & inner vertex $v$ \\
\hline
\end{tabular}

Fig. 3: The closure-bijections, with the parameter correspondences.

erasing all the ingoing half-edges of $M$, thereby creating an undirected embedded bicolored graph with some outgoing stems incident to black corners.

We recall the result from [Ber07] (see also [BC10]) that we shall generalize.

Theorem 3 The rooted closure is a bijection between decorated mobiles of excess $\delta=1$ and (cornerrooted) minimal accessible orientations. The rooted opening is the inverse mapping. Lastly, the parametercorrespondence is shown in Figure 3 top-part.

\subsection{Bijection for $\delta$-mobiles}

$\delta$-closure. We now define the $\delta$-closure of a $\delta$-mobile (the definition depends on the sign of $\delta$ ). Let $D$ be a $\delta$-mobile and let $C$ be the partial closure of $D$. The $\delta$-closure $M$ of $D$ is defined as follows.

- If $\delta>0$, then $C$ has $\delta$ ingoing stems (incident to the root-face). The vertex-rooted orientation $M$ is obtained from $C$ by first creating a root-vertex $v$ of $M$ in the root-face of $C$ and connecting it to each ingoing stem (stems thus become part of an edge of $M$ directed away from $v$ ); second erasing the edges and white vertices of the mobile. 
- If $\delta<0$, then $C$ has $\delta$ outgoing stems (incident to the root-face). The vertex-rooted orientation $M$ is obtained from $C$ by first creating a root-vertex $v$ of $M$ in the root-face of $C$ and connecting it to each outgoing stem and then reorienting these edges (stems thus become part of an edge of $M$ directed away from $v$ ); second erasing the edges and white vertices of the mobile.

- If $\delta=0$, then $M$ is the face-rooted orientation obtained from $C$ by erasing the edges and white vertices of the mobile.

Actually, it is not obvious from our definitions that the $\delta$-closures give connected orientations but we prove this and more below.

Theorem 4 Let $\delta$ be in $\mathbb{Z}$.

- For $\delta>0$, the $\delta$-closure is a bijection between $\delta$-mobiles and the set $\mathcal{S}_{\delta}$, which (by duality) is itself in bijection with the set $\mathcal{O}_{\delta}$. The parameter-correspondence is shown in Figure 3 bottom-part.

- For $\delta<0$, the $\delta$-closure is a bijection between $\delta$-mobiles and the subset $\widetilde{\mathcal{S}}_{|\delta|} \subset \mathcal{S}_{|\delta|}$, which (by duality) is itself in bijection with the subset $\widetilde{\mathcal{O}}_{|\delta|} \subset \mathcal{O}_{|\delta|}$ The parameter-correspondence is shown in Figure 3 bottom-part.

- For $\delta=0$, the $\delta$-closure is a bijection between $\delta$-mobiles and minimal orientations.

The remaining of this section is devoted to the proof of Theorem 4 (the proof for $\delta=0$, which is similar, is omitted since we will not use it in this article).

Case $\delta>0$. We first prove that the $\delta$-closure of a $\delta$-mobile is in $\mathcal{S}_{\delta}$. Let $D$ be a $\delta$-mobile, let $C$ be its partial closure and let $M$ be its $\delta$-closure. As observed above, the mobile $D$ has $\delta>0$ exposed white corners. Let $D^{\prime}$ be the decorated mobile obtained from $D$ by creating a new black vertex $b$, joining $b$ to an exposed white corner, and adding $\delta$ outgoing stems to $b$. The excess of $D^{\prime}$ is 1 , hence by Theorem 3 the rooted closure of $D^{\prime}$ gives a minimal accessible orientation $M^{\prime}$. Moreover, it is easily seen (Figure 4 ) that the root-corner of $M^{\prime}$ is incident to the new vertex $b$ (because the ingoing stem incident to $b$ is not matched during the partial closure). Moreover (provided the ingoing root half-edge is not counted) $b$ is a source of the orientation $M^{\prime}$, and the vertex-rooted orientation $M$ is induced by the corner-rooted orientation $M^{\prime}$. Thus, the orientation $M$ is in $\mathcal{S}_{\delta}$.

The following comment will be useful later (for the case $\delta<0$ ): the closure $M$ of $D$ is in $\widetilde{\mathcal{S}}_{\delta}$ if and only if each of the exposed white corners of $D$ is incident to a (white) leaf of $D$. Indeed, a white vertex $w_{f}$ of $D$ has an exposed white corner if and only if it corresponds to a face $f$ of $M$ incident to the root-vertex $b$. Moreover, the clockwise degree of $f$ is (as always) the degree of $w_{f}$.

We now prove that the $\delta$-closure is a bijection by defining the inverse mapping. Let $M$ be a vertexrooted orientation in $\mathcal{S}_{\delta}$. By applying the partial opening of $M$ and then erasing every ingoing half-edge of $M$, one obtains an embedded graph with stems $\widehat{D}$. The embedded graph $\widehat{D}$ is in fact disconnected since the root-vertex $b$ of $M$ is incident to no edge of $\widehat{D}$ (since $b$ is a source of $M$ ). The $\delta$-opening $D$ of $M$ is obtained from $\widehat{D}$ by erasing the vertex $b$. In order to prove that $D$ is a decorated mobile (i.e. a tree with stems), we consider a minimal accessible orientation $M^{\prime}$ obtained from $M$ by choosing a root-corner for $M$ among the corners incident to the root-vertex $b$. By Theorem 3 , the rooted opening of $M^{\prime}$ gives a decorated mobile $D^{\prime}$. Clearly, $D$ is obtained from $D^{\prime}$ by erasing the black vertex $b$. Moreover, $b$ is a leaf of $D^{\prime}$ (since $b$ is incident to no ingoing half-edge except the stem indicating the root-corner of $\mathrm{O}$ ), hence $D$ is a mobile, and it has excess $\delta$. Lastly, since the rooted closure and rooted opening are inverse mappings, it is clear that $\delta$-closure and $\delta$-opening are inverse mappings, hence bijections. 


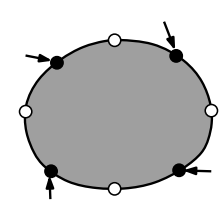

(a)

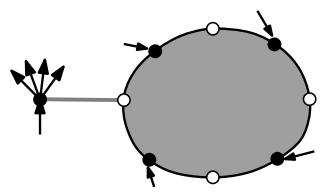

(b)

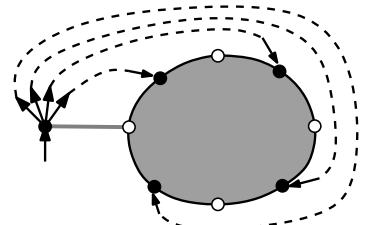

(c)

Fig. 4: Formulation of the $\delta$-closure, for $\delta>0$, as a reduction to the rooted closure. Figure (a) shows generically the partial closure of a $\delta$-mobile with $\delta=4$, in (b) one creates a black vertex $b$ with $\delta$ outgoing stems, and connects it to an exposed white corner, in (c) one performs the remaining matchings of stems to complete the $\delta$-closure.

Case $\delta<0$. We denote $d=-\delta$. Let $D$ be a $\delta$-mobile. We associate to $D$ a $d$-mobile $\phi(D)$ obtained from $D$ by transforming each of its $d$ unmatched outgoing stems into an edge of $\phi(D)$ connected to a newly created white leaf. Observe that the $\delta$-closure of $D$ and the $d$-closure of $\phi(D)$ coincide. Hence the $\delta$-closure is the composition of the mapping $\phi$ and of the $d$-closure. Moreover, the mapping $\phi$ is a bijection between the set of $\delta$-mobiles and the set $\mathcal{D}_{d}$ of $d$-mobiles such that every exposed white corner belongs to a leaf. Indeed, $\phi(D)$ belongs to $\mathcal{D}_{d}$ since the unique incident corner for each of the $d$ newly created white leaves remains exposed during the partial closure; and the inverse mapping $\phi^{-1}$ is obtained by replacing each edge incident to an exposed leaf by an outgoing stem. Lastly, by the observations above (case $\delta>0$ ), the $d$-closure induces a bijection between the set $\mathcal{D}_{d}$ and the set $\widetilde{\mathcal{S}}_{d}$. The inverse mapping to the $\delta$-closure, called the $\delta$-opening, is obtained as the composition of $\phi^{-1}$ with the $d$-opening. This completes the proof of Theorem 4 (in the cases $\delta \neq 0$ ).

\section{Bijective counting of triangulations with boundaries}

In this section we obtain bijections for simple triangulations (a.k.a. 3-connected triangulations, maximal planar graphs) and for triangulations with boundaries. The bijections are obtained by specializing the closures defined in the previous section to certain classes of orientations characterizing simple triangulations.

Let $T$ be a face-rooted triangulation. A 3-orientation of $T$ is an orientation such that inner vertices have indegree 3 and outer vertices have indegree 1. Schnyder proved in [Sch89] that any simple facerooted triangulation admits a 3-orientation, that any 3-orientation is accessible from the outer vertices and that the root-face is always directed. Moreover, one easily checks (using Euler's relation) that loops and double edges are obstructions to the existence of a 3-orientation. Thus, a planar triangulation admits a 3 -orientation if and only if it is simple. In the following we simply call 3-orientation a 3-orientation of a face-rooted triangulation. From Lemma 1 one obtains:

Lemma 5 Face-rooted simple triangulations are in bijection with minimal 3-orientations. Such orientations are clockwise-minimal and accessible.

Minimal 3-orientations are the orientations in $\widetilde{\mathcal{O}}_{3}$ such that all inner vertices have indegree 3 and all faces have degree 3 . Thus, we can use the case $\delta=-3$ of Theorem 4 to conclude that face-rooted simple triangulations are in bijection with (-3)-mobiles having every vertex of degree 3 (recall that outgoing stems count in the degree of a black vertex). In fact, the constraint that the excess is -3 can be omitted, since it is a consequence of all vertices having degree 3 (as easily seen by induction on the number of vertices). Call trivalent the decorated mobiles with all vertices of degree 3 . We obtain: 


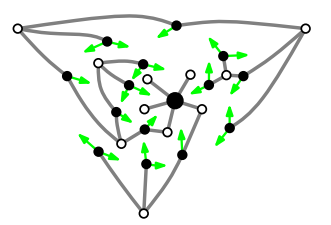

(a)

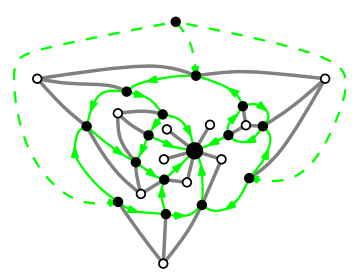

(b)

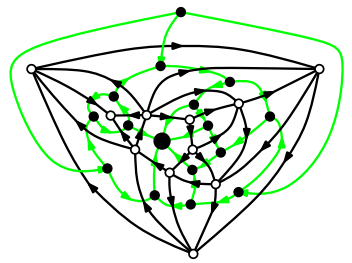

(c)

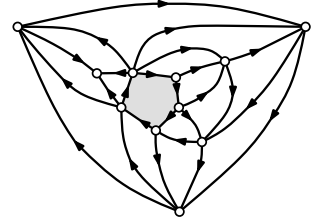

(d)

Fig. 5: (a) A mobile in $\mathcal{B}_{k}$. (b) The 3-closure. (c) Duality. (d) The non-separated k-annular triangulation (with its minimal pseudo 3-orientation).

Proposition 6 (Recovering [FPS08]) The $\delta$-closure, case $\delta=-3$ (together with duality) induces a bijection between face-rooted triangulations with $n+3$ vertices and trivalent mobiles with $n$ white vertices.

Proposition 7 (Counting rooted simple triangulations) For $n \geq 0$, let $t_{n}$ be the number of cornerrooted simple triangulations with $n+3$ vertices. The generating function $T(x)=\sum_{n \geq 0}(2 n+1) t_{n} x^{n}$ satisfies

$$
T(x)=u^{3}, \text { where } u=1+x u^{4} .
$$

Consequently, the Lagrange inversion formula gives: $t_{n}=2 \frac{(4 n+1) !}{(n+1) !(3 n+2) !}$.

Proof: The Euler relation easily implies that a triangulation with $n+3$ vertices has $2 n+1$ non-root faces. Hence $(2 n+1) t_{n}$ is the cardinality of the set $\mathcal{H}_{n}$ of face-rooted triangulations with $n$ inner vertices having an additional marked corner $c$ not incident to the root-face (think of obtaining this map by first marking a corner and then a face). Marking the corner $c$ is equivalent to marking a black corner of the associated mobile (since the black vertices are in correspondence to the triangular faces). In other words, $\mathcal{H}_{n}$ is in bijection, via the $(-3)$-closure, with trivalent mobiles that have $n$ white vertices and a marked black corner, and $T(x)$ is the generating function of this class of mobiles. Finally, the expression of $T(x)$ above is just the translation of a recursive decomposition for the mobiles (details are omitted here).

We now proceed to count bijectively the triangulations with boundaries. In the following, $k$ is an integer greater than 3. A $k$-gonal triangulation is a map having one face of degree $k$ whose contour is simple (incident to $k$ distinct vertices) and all other faces of degree 3 . The $k$-gonal face is called boundary face, and the vertices are called boundary or non-boundary depending on whether they are incident to the boundary face. A pseudo 3-orientation of a $k$-gonal triangulation is an orientation such that all nonboundary vertices have indegree 3 , and the boundary face is directed. A pseudo 3 -orientation of a $k$-gonal triangulation is shown in Figure 5 (d). By the Euler relation, a $k$-gonal triangulation with $n$ non-boundary vertices has $3 n+2 k-3$ edges. Hence, the sum of indegrees of the boundary vertices is $2 k-3$. A $k$-annular triangulation is a face-rooted $k$-gonal triangulation whose root-face is not the boundary face; see Figure 5 d). Let $T$ be a simple $k$-annular triangulation, with root-face $f$ and boundary face $f^{\prime}$. A 3 -cycle $C$ of $T$ is called separating if $C$ is different from (the contour of) the root-face and has $f$ on one side and $f^{\prime}$ on the other side; $T$ is said to be non-separated if it has no separating 3-cycle. We denote by $\mathcal{T}_{k}$ the set of $k$-annular triangulations, and by $\mathcal{N}_{k}$ the subset of non-separated ones.

Lemma 8 A k-annular triangulation A admits a pseudo 3-orientation if and only if it is simple. If $A$ is simple, it admits a unique minimal pseudo 3-orientation. This orientation is accessible from all outer 
vertices if and only if $A$ is non-separated. Moreover, the root-face is directed (in clockwise direction) in this case.

To summarize, the set $\mathcal{N}_{k}$ of non-separated $k$-annular triangulations is in bijection with the set of clockwise-accessible minimal pseudo 3-orientations.

The proof of the lemma, which is ommited, essentially relies on Lemma 1 and on the Euler relation.

By definition, the clockwise-accessible minimal pseudo 3-orientations are the orientations in $\mathcal{O}_{3}$ such that every vertex has indegree 3 and every face has degree 3 except for one boundary face $b$ which has degree $k$ and is directed clockwise. Moreover, a counting argument (using the Euler relation) shows that the indegrees of the boundary vertices must add up to $2 k-3$. Let $D$ be the 3-mobile giving an orientation $O \in \mathcal{N}_{k}$ (by the 3-closure followed by duality) and let $v_{b}$ be the black vertex of $D$ corresponding to the boundary face $b$. Since $b$ is counterclockwise, $v_{b}$ has no outgoing stem and has $k$ white neighbors, which clearly (by definition of closure) corresponds to the boundary vertices; see Figure 5 Hence, the degree of these white vertices of the mobile must add up to $2 k-3$. Lastly, as in the case of triangulations without boundary, the condition of the excess being 3 is implied by the degree conditions on black and white vertices, so can be omitted. To conclude, by specialization of the 3 -closure, Lemma 8 translates into:

Theorem 9 The family $\mathcal{N}_{k}$ of non-separated k-annular triangulations is in bijection with the family $\mathcal{B}_{k}$ of decorated mobiles having every vertex of degree 3 except for one black vertex $b$ of degree $k$ carrying no outgoing stem and such that the degrees of its $k$ (white) neighbors add up to $2 k-3$.

Theorem 10 (Counting rooted $k$-gonal triangulations) Let $k>3, n \geq 0$, and let $t_{k, n}$ be the number of simple corner-rooted $k$-gonal triangulations with $n+k$ vertices having the root-corner in the $k$-gonal face. The generating function $T_{k}(x)=\sum_{n \geq 0}(2 n+k-2) t_{k, n} x^{n}$ satisfies

$$
T_{k}(x)=\left(\begin{array}{c}
2 k-4 \\
k-3
\end{array}\right) u^{2 k-3}, \quad \text { where } u=1+x u^{4} .
$$

Consequently, the Lagrange inversion formula gives: $t_{k, n}=\frac{2(2 k-3) !}{(k-1) !(k-3) !} \frac{(4 n+2 k-5) !}{n !(3 n+2 k-3) !}$.

Proof: Let $\overrightarrow{\mathcal{T}}_{k}$ be the set of $k$-annular triangulations with a marked corner in the boundary face (equivalently, a marked boundary vertex). Let $\overrightarrow{\mathcal{N}}_{k}$ be the subset of these $k$-annular triangulations that are nonseparated. Let also $\overrightarrow{\mathcal{T}}$ be the set of corner-rooted simple triangulations with a marked inner face. The separating 3-cycles of a $k$-annular triangulation are linearly ordered by inclusion of their boundary region (the region which contains the boundary face). Thus, there is a unique decomposition of $k$-annular triangulations $A \in \overrightarrow{\mathcal{T}}_{k}$ into a pair $(N, T) \in \overrightarrow{\mathcal{N}}_{k} \times \overrightarrow{\mathcal{T}}$. This decomposition is a bijection and translates into the generating function equation $T_{k}(x)=N_{k}(x) T(x)$, where $T_{k}(x), N_{k}(x), T(x)$ are respectively the generating function of the maps in $\overrightarrow{\mathcal{T}}_{k}, \overrightarrow{\mathcal{N}}_{k}, \overrightarrow{\mathcal{T}}$ counted by number of non-boundary vertices. The generating function $\overrightarrow{\mathcal{T}}_{k}(x)$ is $\sum_{n \geq 0}(2 n+k-2) t_{k, n} x^{n}$ because maps in $\overrightarrow{\mathcal{T}}_{k}$ with $n$ non-boundary vertices have $2 n+k-2$ non-boundary faces. Moreover, by Proposition 7. $T(x)=u^{3}$, where $u=1+x u^{4}$. It remains to express $N_{k}(x)$ in terms of $u$. By Theorem 9 (and the fact that marking a corner in the boundary face accounts to marking a corner incident to the special black vertex in the associated mobile), $N_{k}(x)$ is the generating function of corner-rooted mobile (counted by number of white vertices) such that the rootvertex is a black corner of degree $k$ whose (white) neighbors have total degree $2 k-3$. The white vertices 
have a total of $k-3$ hanging subtrees, which are trivalent trees whose generating funcion is $v=u^{2}$ (easy proof omitted). In addition, there are $\left(\begin{array}{c}2 k-4 \\ k-3\end{array}\right)$ ways to distribute the $k-3$ hanging trees on the $k$ white vertices. Hence, $N_{k}(x)=\left(\begin{array}{c}2 k-4 \\ k-3\end{array}\right) u^{2 k-6}$.

\section{Bijective counting of quadrangulations with boundaries}

In this section we reiterate the strategy used in previous section to the case of quadrangulations.

We call 2-orientation a face-rooted orientation, in which faces have degree 4 , inner vertices have indegree 2, and outer vertices have indegree 1. De Fraysseix et al. [dFOdM01] have shown that any simple face-rooted quadangulation admits a 2-orientation, that any 2-orientation is accessible from the outer vertices and that the root-face is always directed. Moreover, one easily checks that a double edge is an obstruction for 2-orientation. Hence minimal 2-orientations are in bijection with simple face-rooted quadrangulations. By definition, minimal 2-orientations are the orientations in $\widetilde{\mathcal{O}}_{4}$ having faces of degree 4 and inner vertices of indegree 2 . The (-4)-closure (followed by duality) gives a bijection between such orientations and (-4)-mobiles whise white vertices have degree 2 and whose black vertices have degree 4 . Call these mobiles tetravalent. We obtain:

Proposition 11 (Counting rooted simple quadrangulations) For $n \geq 0$, let $q_{n}$ be the number of rooted simple quadrangulations with $n+4$ vertices. Then the generating function $Q(x)=\sum_{n \geq 0}(n+1) q_{n} x^{n}$ satisfies

$$
Q(x)=u^{4}, \text { where } u=1+x u^{3} .
$$

Consequently, the Lagrange inversion formula gives: $q_{n}=2 \frac{(3 n+3) !}{(n+2) !(2 n+3) !}$.

Call $2 k$-gonal quadrangulation a map with faces of degree 4 except for one face of degree $2 k>4$. The strategy for counting simple $2 k$-gonal quadrangulations parallels the case of triangulations. One first defines a $2 k$-annular quadrangulation to be a simple quadrangle-rooted $2 k$-gonal quadrangulation. One then proves that any such map admits a unique minimal pseudo 2-orientation (orientation such that the boundary face is clockwise and non-boundary vertices have indegree 2), and that this orientation is clockwise-accessible if and only if the map is non-separated (no 4-cycle separates the root-face from the boundary face). Any $2 k$-annular quadrangulation decomposes uniquely into a pair made of a facerooted quadrangulation with an additional marked face and a non-separated $2 k$-annular quadrangulation. Moreover, the later maps are in bijection (via the 4-closure) with a family of pseudo-tetravalent decorated mobiles which is easy to enumerate. We obtain:

Theorem 12 (Counting rooted simple $2 k$-gonal quadrangulations) Let $k>2, n \geq 0$, and let $q_{k, n}$ be the number of rooted simple $2 k$-gonal quadrangulations with $n+2 k$ vertices and with the root-corner incident to the $2 k$-gonal face. The generating function $Q_{k}(x)=\sum_{n \geq 0}(n+k-1) q_{k, n} x^{n}$ satisfies

$$
Q_{k}(x)=\left(\begin{array}{c}
3 k-3 \\
k-2
\end{array}\right) u^{3 k-2}, \text { where } u=1+x u^{3} .
$$

Consequently, the Lagrange inversion formula gives: $\quad q_{k, n}=\frac{3(3 k-2) !}{(k-2) !(2 k-1) !} \frac{(3 n+3 k-4) !}{n !(2 n+3 k-2) !}$. 


\section{References}

[BC10] O. Bernardi and G. Chapuy. A bijection for covered maps, or a shortcut between harerzagier's and jackson's formulas. arXiv:1001.1592, 2010.

[Ber07] O. Bernardi. Bijective counting of tree-rooted maps and shuffles of parenthesis systems. Electron. J. Combin., 14(1):R9, 2007.

[BFG04] J. Bouttier, P. Di Francesco, and E. Guitter. Planar maps as labeled mobiles. Electron. J. Combin., 11(1):R69, 2004.

[BMJ06] M. Bousquet-Mélou and A. Jehanne. Polynomial equations with one catalytic variable, algebraic series and map enumeration. J. Combin. Theory Ser. B, 96(5):623 - 672, 2006.

[Bro64] W.G. Brown. Enumeration of triangulations of the disk,. Proc. London Math. Soc., 14(3):746-768, 1964.

[Bro65] W.G. Brown. Enumeration of quadrangular dissections of the disk. Canad. J. Math., 21:302317, 1965.

[CV81] R. Cori and B. Vauquelin. Planar maps are well labeled trees. Canad. J. Math., 33(5):10231042, 1981.

[dFOdM01] H. de Fraysseix and P. Ossona de Mendez. On topological aspects of orientations. Discrete Math., 229:57-72, 2001.

[Fel04] S. Felsner. Lattice structures from planar graphs. Electron. J. Combin., 11(1), 2004.

[FPS08] É. Fusy, D. Poulalhon, and G. Schaeffer. Dissections, orientations, and trees, with applications to optimal mesh encoding and to random sampling. Transactions on Algorithms, 4(2):Art. 19, April 2008.

[Fus07] É. Fusy. Combinatoire des cartes planaires et applications algorithmiques. PhD thesis, École Polytechnique, 2007.

[GJ83] I. P. Goulden and D. M. Jackson. Combinatorial Enumeration. John Wiley, New York, 1983.

[PS03] D. Poulalhon and G. Schaeffer. A bijection for triangulations of a polygon with interior points and multiple edges. Theoret. Comput. Sci., 307(2):385-401, 2003.

[PS06] D. Poulalhon and G. Schaeffer. Optimal coding and sampling of triangulations. Algorithmica, 46(3-4):505-527, 2006.

[Sch89] W. Schnyder. Planar graphs and poset dimension. Order, 5(4):323-343, 1989.

[Sch98] G. Schaeffer. Conjugaison d'arbres et cartes combinatoires aléatoires. PhD thesis, Univ. Bordeaux I, 1998.

[Tut63] W. T. Tutte. A census of planar maps. Canad. J. Math., 15:249-271, 1963. 